\title{
Antimicrobial activity of cefepime-tazobactam combination tested against clinical isolates of Enterobacteriaceae
}

The Journal of Antibiotics (2014) 67, 603-604; doi:10.1038/ja.2014.45; published online 23 April 2014

Gram-negative pathogens such as members of Enterobacteriaceae are established nosocomial pathogens. ${ }^{1}$ These bacteria have acquired several resistance mechanisms to evade the lethal effect of antibiotics. Extended spectrum $\beta$-lactamases (ESBLs) production is one of the most vital mechanisms of resistance against $\beta$-lactam antibiotics. $^{2}$ In India, the ESBL-producing strains are highly prevalent, making majority of the available antibiotics ineffective. Consequently, we are left with very few treatment options, including $\beta$-lactam and $\beta$-lactamase inhibitor (BL-BLI) combinations, carbapenems and colistin. BL-BLI combinations inhibit the $\beta$-lactamase enzyme and thus have activity against ESBLs but their role in severe infections caused by ESBLs is not reliable. ${ }^{2}$ In addition to that, resistance to carbapenems and colistin has also been observed to be mounting up recently leading to a world without antibiotics. ${ }^{3,4}$

All this has compelled the researchers to find newer agents and to generate newer combinations with already available antibiotics. Cefepime, the fourth-generation cephalosporin, has an extended spectrum of activity against Gram-negative pathogens. It is stable against both AmpC and OXA, but lacks activity against ESBLs, ${ }^{5}$ while tazobactam is active against ESBLs. ${ }^{6}$ Thus, a novel combination of cefepime-tazobactam (CPT) is expected to increase susceptibility of Enterobacteriaceae, by effectively covering all the three major mechanisms of resistance (ESBL, AmpC and OXA). The present study was carried out in an 1800 bedded tertiary care super-speciality teaching hospital in North India to assess the in vitro activity of $\mathrm{CPT}$ and to compare it with various other routinely used antibiotics. This study is a part of the ongoing 'ASCENT' study, which is a study of in vitro sensitivity of cefepimetazobactam and other antimicrobial agents against enterobacteriaceae isolated from hospitalized patients of a tertiary care hospital in India. A total of 500 consecutive, non-duplicate isolates of Escherichia coli (167), Klebsiella pneumoniae (250), Enterobacter aerogenes (48), Proteus mirabilis (25) and Citrobacter spp. (10) isolated from various clinical specimens, blood (408), pus (74), body fluids (08), respiratory (07) and cerebrospinal fluid (03) between August 2012 and March 2013, were included in the study. These isolates were identified by standard conventional methods and antibiotic susceptibility testing was performed by Kirby Bauer disk diffusion method as per Clinical and Laboratory Standards Institute (CLSI), 2013. ${ }^{7}$ Owing to the lack of CLSI interpretive criteria for cefoperazone-sulbactam (CSL) and CPT, we have used the zone interpretative criteria for cefoperazone and cefepime, respectively. E. coli ATCC 25923 was used as the control. The strains were tested for ESBL production according to CLSI guidelines, 2013 and for AmpC- $\beta$-lactamase production by EDTA disc test. ${ }^{7,8}$

The prevalence of ESBL-producing strains in India is very high. ESBL rate in our study was also found to be $60 \%$, which is comparable to the other studies. The prevalence of ESBLs in India has now reached epidemic proportions, ranging from 62 to $100 \%$ in E. coli and Klebsiella spp. isolated from various clinical samples as was observed from 10 Indian medical centers as part of SENTRY study (a global ongoing surveillance for monitoring antimicrobial resistance). ${ }^{9}$ In our study, as shown in Table 1, carbapenems were found to be the most effective drugs (imipenem more effective than meropenem) for all the isolates. In routine, these agents are considered to be the last resort to combat infections by multidrug-resistant bacteria, especially in intensive care units and high-risk wards. In contrast, the third-generation cephalosporins (ceftazidime and cefotaxime) were found to be the least effective for all the isolates. CPT was only next to carbapenems with high susceptibility against E. coli, E. aerogenes and P. mirabilis. However, it was not found to be much effective against $K$. pneumoniae with only $36 \%$ isolates being susceptible to it. This drug combination also gave better results for all the isolates when compared to either cefepime or piperacillin-tazobactam (PTZ) used alone. Among the BL-BLI combinations (PTZ, CSL and CPT) tested, CPT displayed

Table 1 Comparison of the susceptibility of CPT to various other antibiotics (in percentage)

\begin{tabular}{lrrrrrrrr}
\hline Organism (no. of isolates) & IPM & MER & FEP & CAZ & CTX & PTZ & CSL & CPT \\
\hline Escherichia coli (167) & 94 & 53 & 22 & 13 & 12 & 58 & 63 & 75 \\
Klebsiella pneumoniae (250) & 86 & 54 & 19 & 12 & 13 & 35 & 33 & 36 \\
Enterobacter aerogenes (48) & 98 & 67 & 29 & 21 & 21 & 46 & 50 & 58 \\
Proteus mirabilis (25) & 100 & 70 & 56 & 40 & 52 & 80 & 70 & 88 \\
\hline
\end{tabular}

Abbreviations: CAZ, ceftazidime; CPT, cefepime-tazobactam; CSL, cefoperazone-sulbactam; CTX, cefotaxime; FEP, cefepime; IPM, imipenem; MER, meropenem; PTZ, piperacillin-tazobactam. 
better susceptibility for the study isolates. Similar results were observed by $\operatorname{Sood}^{10}$ also, whereby, among six BL-BLI combinations tested against Enterobacteriaceae, CPT was found to be the best combination with sensitivity of $90.6 \%$, followed by CSL (84.9\%) and PTZ (54\%). In vitro susceptibility results of our study suggest that CPT can act as a carbapenem saving antibiotic as repeated exposure to carbapenem has its own cost in terms of emergence of other multi-resistant organisms. $^{11}$

As only limited number of studies are available for this agent, extensive studies are required to establish breakpoint levels for susceptible and resistant categories and to further verify the activity of this novel antibiotic combination.

\section{ACKNOWLEDGEMENTS}

This study was funded by AstraZeneca Pharma India Limited, Bangalore 560024, India.
Ramanpreet Kaur ${ }^{1}$, Vikas Gautam ${ }^{1}$, Lipika Singhal ${ }^{2}$ and Pallab Ray ${ }^{1}$

${ }^{1}$ Department of Medical Microbiology, PGIMER, Chandigarh, India and ${ }^{2}$ Department of Microbiology, Government Medical College and Hospital, Chandigarh, India

E-mail:r_vg@yahoo.co.uk

1 Hawser, S. P. et al. Emergence of high levels of extended-spectrum-beta-lactamase-producing gramnegative bacilli in the Asia-Pacific region: data from the Study for Monitoring Antimicrobial Resistance Trends (SMART) program, 2007. Antimicrob. Agents Chemother. 53, 3280-3284 (2009).

2 Rawat, D. \& Nair, D. Extended-spectrum betalactamases in Gram Negative Bacteria. J Glob. Infect. Dis. 2, 263-274 (2010)

3 Falagas, M. E. et al. Outcome of infections due to pandrug-resistant (PDR) Gram-negative bacteria. BMC Infect. Dis. 5, 24 (2005).

4 Marchaim, D. et al. Outbreak of colistin-resistant, carbapenem-resistant Klebsiella pneumoniae in metropolitan Detroit, Michigan. Antimicrob. Agents Chemother. 55, 593-599 (2011).

5 Endimiani, A., Perez, F. \& Bonomo, R. A. Cefepime: a reappraisal in an era of increasing antimicrobial resistance. Expert. Rev. Anti. Infect. Ther. 6, 805-824 (2008).

6 Rupp, M. E. \& Fey, P. D. Extended spectrum beta-lactamase (ESBL)-producing Enterobacteriaceae: considerations for diagnosis, prevention and drug treatment. Drugs 63, 353-365 (2003).

7 Performance standards for antimicrobial susceptibility testing; Twentieth informational supplement. in Clinical and Laboratory Standards Institute (CLSI), M100-S23 Vol. 30. (CLSI, Wayne, PA, 2013).

8 Black, J. A., Moland, E. S. \& Thomson, K. S. AmpC disk test for detection of plasmid-mediated AmpC beta-lactamases in Enterobacteriaceae lacking chromosomal AmpC beta-lactamases. J. Clin. Microbiol. 43, 3110-3113 (2005).

9 Manoharan, A., Premalatha, K., Chatterjee, S. \& Mathai, D. Correlation of TEM, SHV and CTX-M extended-spectrum beta lactamases among Enterobacteriaceae with their in vitro antimicrobial susceptibility. Indian J. Med. Microbiol. 29, 161-164 (2011).

10 Sood, S. Comparative evaluation of the in-vitro activity of six beta-lactam/beta-lactamase inhibitor combinations against Gram negative bacilli. J. Clin. Diagn. Res. 7, 224-228 (2013).

11 Al-Muharrmi, Z., Rafay, A., Balkhair, A. \& Jabri, A. A. Antibiotic combination as empirical therapy for extended spectrum Beta-lactamase. Oman Med. J. 23, 78-81 (2008). 